\title{
Single-molecule imaging reveals topology dependent mutual relaxation of polymer chains
}

Maram Abadi ${ }^{\ddagger}$ Maged F. Serag ${ }^{\ddagger}$, and Satoshi Habuchi ${ }^{*}$

Biological and Environmental Sciences and Engineering Division, King Abdullah University of

Science and Technology, P.O. Box 4700 KAUST, Bldg 2 Room 4277, Thuwal 23955-6900,

Saudi Arabia. 
ABSTRACT: The motion and relaxation of linear and cyclic polymers under entangled conditions are investigated by means of newly developed single-molecule tracking technique, cumulativearea (CA) tracking. CA tracking enables simultaneous quantitative characterization of the diffusion mode, diffusion rate, and relaxation time that have been impossible with widely-used conventional single-molecule localization and tracking method, by analyzing cumulative areas occupied by the moving molecule. Using the novel approach, we investigate the motion and relaxation of entangled cyclic polymers, which have been an important but poorly understood question. Fluorescently-labeled $42 \mathrm{kbp}$ linear or cyclic tracer dsDNAs in concentrated solutions of unlabeled linear or cyclic DNAs are used as model systems. We show that CA tracking can explicitly distinguish topology-dependent diffusion mode, rate, and relaxation time, demonstrating that the method provides an invaluable tool for characterizing topological interaction between the entangled chains. We further demonstrate that the current models proposed for the entanglement between cyclic polymers which are based on cyclic chains moving through an array of fixed obstacles cannot correctly describe the motion of the cyclic chain under the entangled conditions. Our results rather suggest the mutual relaxation of the cyclic chains, which underscore the necessity of developing a new model to describe the motion of cyclic polymer under the entangled conditions based on the mutual interaction of the chains. 


\section{INTRODUCTION}

The motion of entangled polymer chains continues to be an important research topic in polymer science. ${ }^{1-5}$ The diffusive motion and relaxation of individual chains under entangled conditions are the major factors for controlling the rheological properties of polymers, and thus they serve as a basis for the development of polymer materials such as thin films and fibers. Extensive studies over the decades have demonstrated that the motion of long linear polymers can be interpreted by the reptation model in which a molecule moves along a virtual tube created by surrounding molecules. ${ }^{1-3}$ In the reptation model, the motion of the chain ends governs the motion of the entire chain. On the other hand, the motion of topologically-unique cyclic polymers under the entangled conditions should be distinct from that of the linear counterparts by the absence of the chain ends. ${ }^{6}$ Indeed, cyclic polymers frequently show unexpected behaviors that are distinct from the linear counterparts. For instance, recent studies demonstrated that thermal stability of polymer micelles increased drastically by a linear-to-cyclic conversion. ${ }^{7}$ ${ }^{8}$ Simulation studies have also suggested an essential role of a loop formation of DNA in a spatial organization of chromosomes. ${ }^{9-13}$ These studies point to the critical roles of the cyclization of the polymer chains in various research fields, from materials science to life science.

Several models have been proposed to describe the motion of cyclic polymers. Cyclic chains are described by double-folded linear conformations in the double-folded reptation model, whereas cyclic chains adopt double-folded linear conformations with multiple loops in the lattice-animal model. ${ }^{14,15}$ In these models, cyclic chains move through an array of fixed obstacles (i.e., single chain model), similar to the reptation theory. However, the applicability of these models has been questioned. Recent simulation studies suggest that the motion of entangled cyclic polymers is better described as a single cyclic chain moving through an array of non-fixed obstacles due to 
the mutual relaxation effect. ${ }^{16}$ The motion of the chains in linear-cyclic blends has been reported to be even more complicated. ${ }^{17,18}$ Despite decades of experimental ${ }^{19-23}$ and simulation studies, ${ }^{24-}$ ${ }^{27}$ the motion of entangled cyclic polymers remains controversial because the concept and the role of the entanglement between the cyclic polymers has not been clarified. ${ }^{28}$ This is partly due to the lack of a method that can directly characterize the motion of individual polymer chains in an entangled melt and solution under equilibrium conditions.

Static and dynamic properties of polymers have been characterized by ensemble-averaged methods such as NMR, light scattering, viscosity, and stress relaxation measurement. ${ }^{29-31}$ Although the current polymer physics theory has been developed based on the findings obtained by those methods, molecular mechanisms that governs the entangled polymer dynamics remain to be elucidated. This is due to the inherent conformational heterogeneity of polymers. Singlemolecule imaging studies have been revealing the spatiotemporal heterogeneity of polymer dynamics by visualizing the motion of individual polymer chains ${ }^{32-36}$ or small fluorophores embedded in polymer matrices. ${ }^{37-44}$ Using single-molecule localization and tracking (SMLT) technique, we previously reported the multimode diffusion of a cyclic and dicyclic polymers under the entangled conditions by incorporating a fluorophore into the chains. ${ }^{17,45,46}$ These studies have suggested a direct connection between the conformational state of the chains and the diffusive motion. The motion and conformational relaxation of single polymer chains under the entangled conditions have been studied by using fluorescently-labeled micrometers-long molecules, typically dsDNA molecules. ${ }^{47-50}$ The diffusion rates, ${ }^{48,49}$ modes of the chain motion (e.g. reptation motion), ${ }^{47}$ and the conformational relaxation of the chain ${ }^{51,52}$ have been characterized by SMLT combined with molecular manipulation techniques (e.g. optical tweezers). However, these studies have been conducted under non-equilibrium conditions using 
external forces. Further, the diffusive motion has been characterized at the length scale much larger than the size of the molecule because SMLT does not work well for long and flexible molecules at the length scale of the molecule due to the large localization error, ${ }^{49}$ although the diffusive motion within the length scale of the molecule and the relaxation dynamics under the equilibrium conditions most reflect the chain interactions under the entangled conditions. The anti-Brownian electrokinetic trap method has been used for the simultaneous characterization of the molecular motion and relaxation. ${ }^{53,54}$ In this technique, the measurement has to be conducted in the presence of external force, and the diffusion rates and modes are deduced from the applied force. Thus, the analysis of a non-random motion is very difficult. Fluorescence correlation spectroscopy (FCS) can also report the rate of the center-of-mass diffusion and the local relaxation of long and flexible molecules ${ }^{55-58}$ Due to the principle of the technique, the motion of a single molecule cannot be followed over a long time period with this approach, which makes it difficult to apply the method to analyze the mode of diffusive motion. The limitations of the current single-molecule methods point to the necessity of developing a new single-molecule technique which allows for a simultaneous characterization of molecular motion at the length scale of the chain and conformational dynamics at the equilibrium conditions.

We have recently developed a new single molecule fluorescence imaging technique, cumulativearea (CA) tracking, ${ }^{59}$ which allows simultaneous characterization of the diffusion rate and conformational relaxation time of individual polymer molecules in a diluted solution. As compared with the conventional single-molecule fluorescence imaging technique based on SMLT, CA tracking is especially effective in the analysis of the motion of long polymer chains because the center-of-mass diffusion at the length scale of the chain and conformational 
relaxation of the molecule under the equilibrium conditions are characterized accurately by analyzing cumulative areas occupied by the moving molecule.

In this study, we further develop the CA tracking technique such that diffusion modes under entangled conditions can also be characterized together with the diffusion rate and relaxation time. Using the novel CA approach, we investigate the molecular mechanism of the motion of cyclic polymers in an entangled solution at the single molecule level. We used $42 \mathrm{kbp}$ linear and cyclic dsDNA whose contour length is $19 \mu \mathrm{m}$ as model polymers. ${ }^{60}$ DNA has several advantages over synthetic polymers frequently used in rheology experiments, including the guaranteed high purity of the linear and cyclic molecules (synthetic polymers are often impure), ${ }^{61}$ the identical length of all the molecules, and the simple fluorescence labeling of the molecule. These factors make DNA an especially good model system for studying entanglement between polymer chains. We investigate the motion of tracer DNAs in a concentrated solution of unlabeled DNAs (Fig. 1). The modes and rates of the diffusive motion and relaxation times of the entangled linear and cyclic DNA are explicitly distinguished by CA tracking, demonstrating that the method provides an invaluable tool for the direct visualization of topological interactions between polymer chains. Our results show that neither the double-folded model nor the lattice-animal model describe correctly the motion of the cyclic chain under the entangled conditions. Instead, the results suggest the mutual relaxation of the cyclic chains, which underscore the necessity of developing a new model based on the mutual interaction of the chains. 


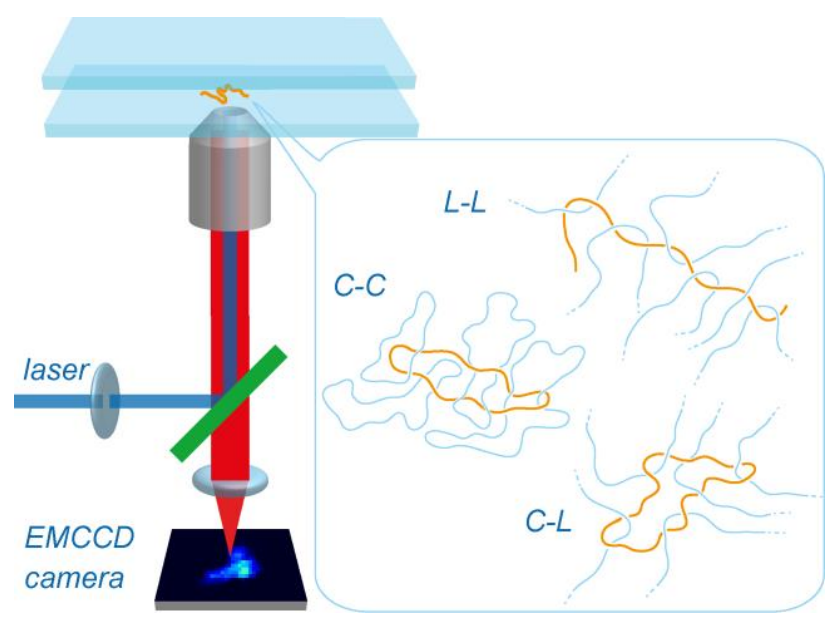

Figure 1. Schematic illustration of the single-molecule fluorescence imaging setup for direct visualization of the motion of individual polymer chains in an entangled solution.

\section{EXPERIMENTAL SECTION}

\section{Sample preparations.}

Charomid (42 kbp) DNA was purchased from Nippon Gene. The linear and cyclic forms of Charomid DNA were prepared from the supercoiled form of the corresponding DNA using SMaI and Topoisomerase I, respectively. To prepare the fluorescently labeled DNA, we dissolved linear and cyclic DNA samples in Tris-EDTA (TE) buffer at the concentration of $5 \mu \mathrm{g} \mathrm{ml}{ }^{-1}$. A 3 $\mu \mathrm{l}$ of TE buffer containing the DNA intercalator dye, YOYO-I $(10 \mu \mathrm{M})$ and a $20 \mu \mathrm{l}$ of DNA solution were mixed with $977 \mu \mathrm{l}$ of TE buffer. This gave a DNA concentration of $1 \mu \mathrm{g} \mathrm{ml}^{-1}$ with a base pair (bp) : dye molecule ratio of $50: 1$. A dilute solution of fluorescently labeled linear (Dilute L) and cyclic (Dilute C) DNA was prepared by adding $20 \mu \mathrm{l}$ of the fluorescently labeled DNA ( $1 \mu \mathrm{g} \mathrm{ml-1)} \mathrm{into} 180 \mu \mathrm{l}$ of TE buffer, which gave a final DNA concentration of $0.1 \mu \mathrm{g} \mathrm{ml^{-1 }}$. 
For the preparation of the concentrated DNA solutions, a $1 \mu$ of the fluorescently labeled tracer DNA $(1 \mu \mathrm{g} \mathrm{ml}-1)$ and a $0.5 \mu \mathrm{l}$ of $1 \mathrm{M} \mathrm{NaCl}$ solution were added into a solution of the matrix DNA ( $8.5 \mu$ c containing $10 \mu \mathrm{g}$ of DNA). The solution was gently mixed. The final concentrations of the fluorescently labeled tracer and the non-labeled matrix DNA were $0.1 \mu \mathrm{g}$ $\mathrm{ml}^{-1}$ and $1 \mathrm{mg} \mathrm{ml}^{-1}$, respectively. We prepared three different combinations of the tracer and the matrix DNA: linear tracer DNA in the linear matrix DNA (L-L), cyclic tracer DNA in the cyclic matrix DNA (C-C), and cyclic tracer DNA in the linear matrix DNA (C-L) (Fig. 1). The $10 \mu \mathrm{l}$ DNA sample was sandwiched between a clean coverslip and a glass slide. The sample was sealed by a double-sided adhesive (Grace-Biolabs). The adhesive also served as a spacer and provided the sample thickness of $0.12 \mathrm{~mm}$ (see the Supporting Information).

\section{Single molecule fluorescence imaging.}

The single-molecule fluorescence imaging experiments were performed with a home-built fluorescence microscopy setup that consisted of an inverted microscope (IX71, Olympus) and an EM-CCD camera (iXon Ultra 897, Andor Technology). ${ }^{59}$ The sample was illuminated by a 488 nm line of a continuous wave (CW) solid state laser (MLD, Cobolt) through an objective lens (UAPON 100XO TIRF NA 1.49 oil immersion or UPLSAPO 60 XW NA 1.2 water immersion). The excitation power was adjusted to $0.9 \mathrm{~W} \mathrm{~cm}^{-2}$. The exposure of the EM-CCD camera was synchronized with the sample illumination by an acousto-optical tunable filter (AOTF; AA Optoelectronics) using a laser control system (PCUB-110, Andor Technology) to minimize photo-damage to the sample. The fluorescence from the sample was collected by the same objective and was focused on the EM-CCD camera. When the fluorescence images were recorded with the $\times 60$ objective, an additional lens with $\times 1.6$ magnification was inserted in the 
detection pass. Fluorescence images were recorded with frame rates between $156 \mathrm{~Hz}$ and $0.4 \mathrm{~Hz}$. The frame rates were determined based on the diffusion rates of the molecules so that the diffusion constants could be determined most accurately using the cumulative-area tracking method. The pixel size of the images recorded by the $\times 100$ and $\times 60$ objectives were 160 and 167 $\mathrm{nm}$, respectively (see Supporting Information). The CA tracking analysis was applied only to the molecules from which we were able to obtain diffusion trajectories longer than 100 frames. We also applied the rule that the tracked molecule did not escape from the focal plane in more than one percent of the total frames. These conditions guaranteed the accuracy of the CA tracking analysis. ${ }^{59}$

\section{Determination of the diffusion coefficients by cumulative-area (CA) tracking.}

The diffusion coefficients of the DNA molecules were determined by CA tracking analyses of the fluorescence images obtained by the singe molecule imaging experiments.$^{59} \mathrm{We}$ first defined five image pixels that corresponded to the area occupied by the molecule in each frame. Next, we calculated the cumulative area occupied by the diffusing molecule at the time point $t=t_{i}$ by superimposing all the images obtained in the previous step from $t=t_{1}$ until $t=t_{\mathrm{i}}$. Then, the increments of the cumulative areas due to the diffusion of the molecule (i.e., cumulative area difference (CAD)) that occurred between the time frame $t_{i}$ and $t_{i+1}, t_{i}$ and $t_{i+2}, t_{i}$ and $t_{i+3}, \ldots \ldots, t_{i}$ and $t_{i+n}$, were calculated. Occasionally, the CAD dropped to zero because the newly occupied area was masked by the already occupied cumulative area. In this case, the CAD was determined by reversing the order of the image superimposition. The mean CADs (MCADs) were calculated using the cumulative area differences obtained in the previous step and plotted against the time lag $\left(\Delta t=t_{i+n}-t_{i}\right)$. The diffusion coefficient $(D)$ was calculated by the initial slope of the CAD 
plot (see Supporting Information). The diffusion coefficient was also calculated using the conventional LT method by mean squared displacement analysis described previously. ${ }^{17,46}$

\section{Simulation of $1 D$ and 2D random diffusion.}

A nonrandom diffusive motion is expected for entangled semi-flexible chains. Thus, the diffusion trajectories for the 1D and 2D random motion were constructed using routines written in Matlab. The generated displacements in each time step followed a probability distribution function for the random diffusion expected from the normal diffusion theory. ${ }^{62}$ We generated random values between 0 and $2 \pi$ and the value 0 or $\pi$ for the angles between the two consecutive displacements for the 2D random and 1D random diffusion trajectories, respectively. Based on the positions of the molecule in each simulated trajectory, the locations of the five pixels representing the area occupied by the molecule were determined. Using the simulated trajectories, the diffusion coefficients were calculated by CA tracking analysis (see the Supporting Information).

\section{Analysis of the diffusion mode by CA tracking.}

The diffusion mode was analysed based on the time course of the cumulative area obtained from the image analysis described above. A mean cumulative area (MCA) was calculated using the cumulative area data obtained from approximately 100 molecules. The MCAs for the simulated 1D and 2D random diffusions were calculated using 100 simulated trajectories. To compare the MCAs obtained experimentally with those obtained from the simulated trajectories, we set the average displacements in the simulated $1 \mathrm{D}$ and $2 \mathrm{D}$ random trajectories to the values equal to the mean displacements obtained experimentally. The diffusion modes were analyzed by comparing the scaling exponents obtained for the experimentally determined MCAs with those obtained for 
the simulated MCAs by fitting the MCAs with a power-law function (i.e., different diffusion modes were distinguished by the slope of the double logarithmic MCA plot). A change in the slope of the double logarithmic MCA plot suggests to the presence of different diffusion modes at different length scale. In such cases, the MCAs were analyzed by fitting each regime separately (see the Supporting Information).

\section{Determination of the relaxation time by $\mathrm{CA}$ tracking.}

The conformational relaxation time of the DNA molecules was determined by analyzing the temporal fluctuation of the area occupied by the molecule. ${ }^{59}$ The area occupied by the molecule in this analysis was determined by setting the thresholds in such a way that all parts of the molecule were included when the molecule adopted a fully extended conformation. The relaxation time $\left(\tau_{\mathrm{r}}\right)$ was estimated by fitting the autocorrelation curve of the temporal fluctuation of the area occupied by the molecule using a single-exponential decaying function (see the Supporting Information).

\section{Analysis of the heterogeneity of the diffusion rate.}

The distributions of the diffusion coefficient obtained by CA tracking analysis $\left(\sigma_{D}\right.$; standard deviation of the frequency histogram) were quantitatively characterized by comparing with those obtained from the simulated $2 \mathrm{D}$ random diffusion trajectories $\left(\sigma_{D, \text { sim }}\right)$. The deviation from the homogeneous diffusion was evaluated by the ratio between $\sigma_{D}$ and $\sigma_{D \text {,sim. }}$ A larger $\sigma_{D} / \sigma_{D \text {,sim }}$ value corresponds to the presence of heterogeneous diffusion rates of individual molecules in the sample (see the Supporting Information).

\section{RESULTS AND DISCUSSION}




\section{Modes of the diffusive motion of entangled chains.}

Figure 2 shows time-lapse fluorescence images obtained for L-L (Fig. 2a top), C-C (Fig. 2b top), and C-L (Fig. 2c top). Previous studies on dsDNA with similar length indicate that the motion of L-L is described reasonably by the tube-like reptation motion at the concentration similar to the present study (i.e., $0.8-1.0 \mathrm{mg} \mathrm{ml}^{-1}$ ). The time-lapse images in Figure 2 showed topologydependent diffusion motion and conformational relaxation of the tracer molecules. While L-L displayed directional motion at the length scale of the molecule, more random motion was observed for $\mathrm{C}-\mathrm{C}$ and $\mathrm{C}-\mathrm{L}$. The mode of the diffusive motion was quantified by analyzing the cumulative area occupied by the moving tracer molecules (Fig. 2a bottom, 2b bottom, 2c bottom). We simulated the one-dimensional (1D) and two-dimensional (2D) random motion of the molecule and calculated the cumulative area occupied by the molecule (see Experimental Section, Fig. S1). The 1D random motion corresponds to the reptation and the double-folded reptation motion. The $2 \mathrm{D}$ random motion corresponds to either the random Brownian motion or the lattice-animal motion. The mean cumulative area (MCA) vs time plots obtained from 100 simulated trajectories are displayed in Figure 3 together with experimentally obtained MCA plots (see Experimental Section, Figs. S2 - S6). The diffusion modes were distinguished by the exponents obtained by a power-law fitting of the MCA plots (i.e., the slope of the plots in Fig. 3) (see Experimental Section, Fig. 3 and Table S1). The experimentally obtained MCA plots for the dilute DNA are reasonably reproduced by the 2D random model (Figs. 3a, S2, and S3) irrespective of its topology. This is consistent with the random Brownian motion of the molecules in the dilute solution.

In contrast, the topology of the molecule contributes largely to the diffusion mode in the entangled solution. The MCA plot of L-L shows $1 \mathrm{D}$ random motion up to $\sim 3 \mu \mathrm{m}^{2} \mathrm{MCA}$ (Figs. 
$3 \mathrm{~b}$ and $\mathrm{S} 4$ ), which can be interpreted by the reptation model. While 2D random motion of the center-of-mass at the length scale of the molecule is expected by the reptation theory for an ideal flexible polymer in the limit of long chain, the dsDNA used in this study has been regarded as a semi-flexible chain. ${ }^{63}$ This leads to nonrandom orientations of the chain ends and therefore results in the anisotropic 1D motion of the chains. ${ }^{64}$ This finding is consistent with a previous study that reported the number of entanglements per chain $\left(N_{\mathrm{e}}\right)$ to be about $N_{\mathrm{e}}=7$ for dsDNA of a similar length $(\lambda$-DNA, $48.5 \mathrm{kbp})$ at $0.8 \mathrm{mg} / \mathrm{ml}$ concentration, suggesting reptation motion of the molecule. ${ }^{65}$ When the MCA is greater than $\sim 3 \mu \mathrm{m}^{2}$, the slope of the MCA plot is close to that of $2 \mathrm{D}$ random motion (Fig. 3b), which is also predicted for reptation motion at a length scale larger than the size of the molecule. ${ }^{49}$ The transition between the two diffusion regimes is roughly consistent with the square end-to-end distance $\left(\sim 2.5-3.2 \mu \mathrm{m}^{2}\right)$ of the molecule, ${ }^{66}$ which supports the suggestion that we can distinguish between diffusion modes at different length scales based on the slope of the MCA plot. As described above, the modes of diffusion within the length scale of the molecule most reflect the specific chain interactions. Importantly, the data presented in Figure $3 b$ unequivocally demonstrates that the diffusion modes at that length scale can be quantitatively characterized by CA tracking, which is currently impossible with other single-molecule tracking techniques.

The experimentally obtained MCA plot of C-C shows a slope between the 2D and 1D random motion of up to $\sim 2 \mu \mathrm{m}^{2} \mathrm{MCA}$ (Figs. 3c and S5), which corresponds roughly to the calculated squared end-to-end distance of the molecule. The observation cannot be interpreted as either double-folded reptation motion (i.e., 1D motion) or lattice-animal motion (i.e., 2D motion due to the random motion of the center-of-mass), which have been previously proposed to describe the motion of long flexible cyclic polymers in an entangled melt or solution (see below) ${ }^{67-69}$ The 
anisotropic motion of the cyclic molecule in C-C suggested by the MCA plot could reflect an anisotropic conformation of cyclic chains in a concentrated solution, which was predicted by a recent simulation study. ${ }^{26}$ When the MCA is greater than $\sim 2 \mu \mathrm{m}^{2}$, the slope of the MCA plot is close to that of $2 \mathrm{D}$ random motion (Fig. 3c). The experimentally obtained MCA plot of C-L is reasonably reproduced by $2 \mathrm{D}$ random motion (Figs. $3 \mathrm{~d}$ and $\mathrm{S6}$ ). While the diffusion mode cannot be precisely determined by this result, our observation clearly demonstrates the isotropic motion of the cyclic molecule in the entangled solution of the linear molecule at the length scale of the molecule. This together with its diffusion rate provides a clue about the entanglement between the cyclic and linear chains (see below). 

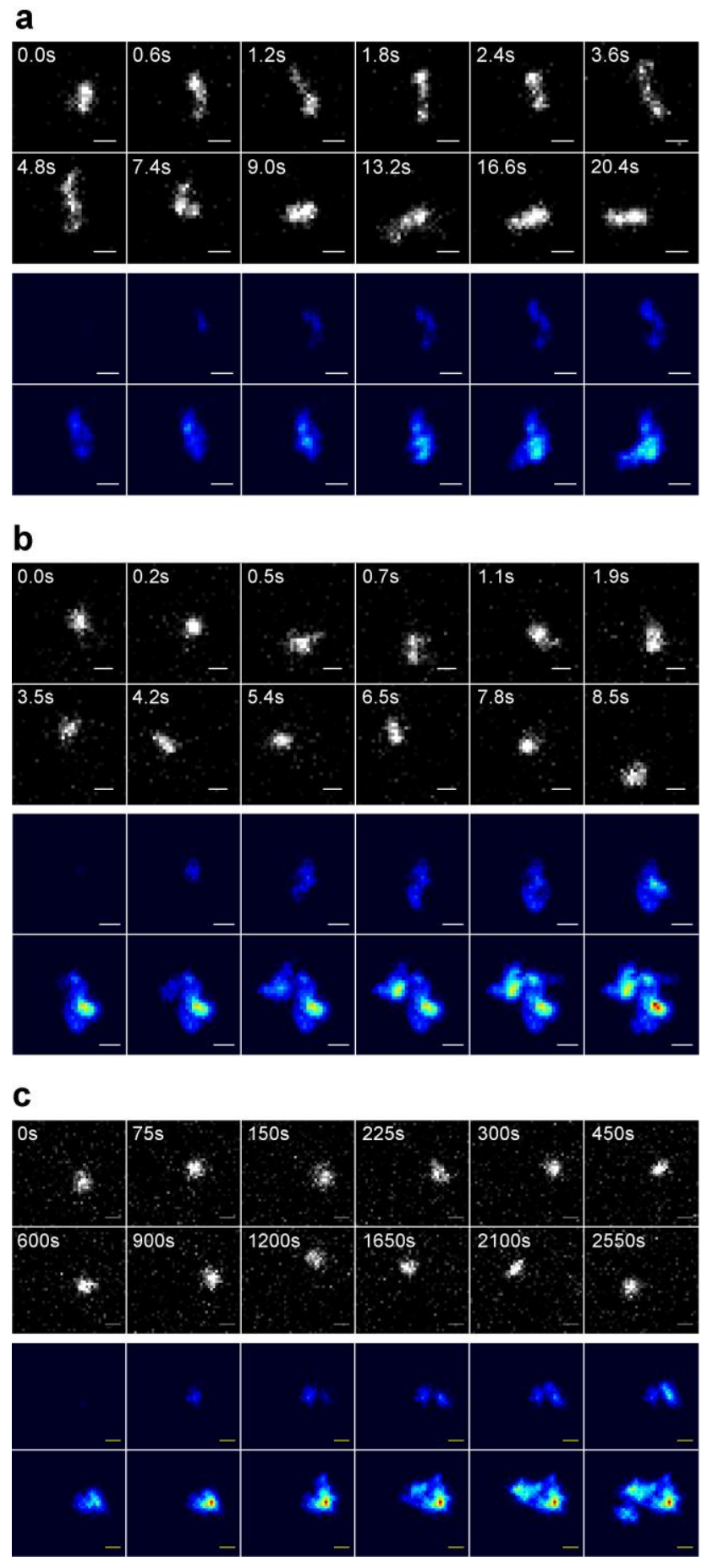

Figure 2. Time lapse fluorescence images and CA tracking images. (Top) Time lapse fluorescence images of (a) a fluorescently labeled $42 \mathrm{kbp}$ linear tracer DNA in a $1 \mathrm{mg} / \mathrm{ml}$ solution of unlabeled $42 \mathrm{kbp}$ linear DNA (L-L); (b) a fluorescently labeled $42 \mathrm{kbp}$ cyclic tracer 
DNA in a $1 \mathrm{mg} / \mathrm{ml}$ solution of unlabeled $42 \mathrm{kbp}$ cyclic DNA (C-C); and (c) a fluorescently labeled $42 \mathrm{kbp}$ cyclic tracer DNA in a $1 \mathrm{mg} / \mathrm{ml}$ solution of unlabeled $42 \mathrm{kbp}$ linear DNA (C-L). (Bottom) The time lapse images of the cumulative area occupied by (a) the moving, fluorescently labeled linear tracer DNA; (b) the moving, fluorescently labeled cyclic tracer DNA; and (c) the moving fluorescently labeled cyclic tracer DNA displayed in the top panels.

Scale bars $=1 \mu \mathrm{m}$. 
a

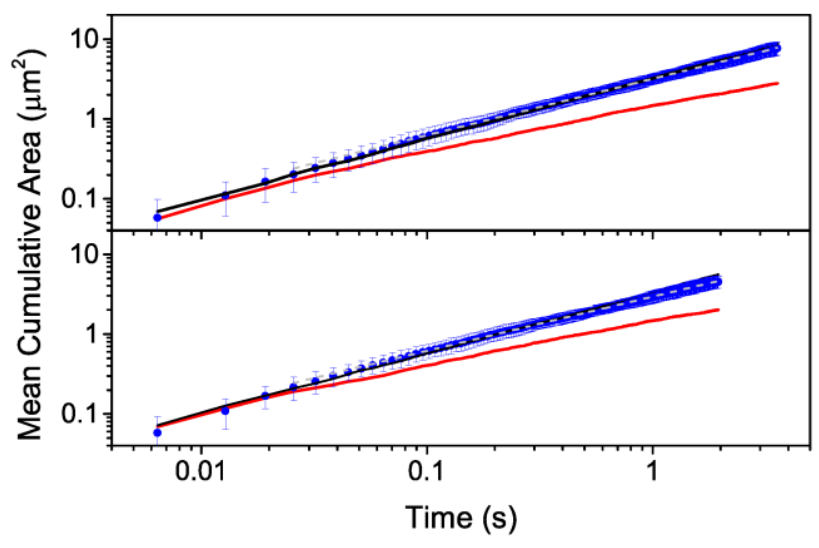

C

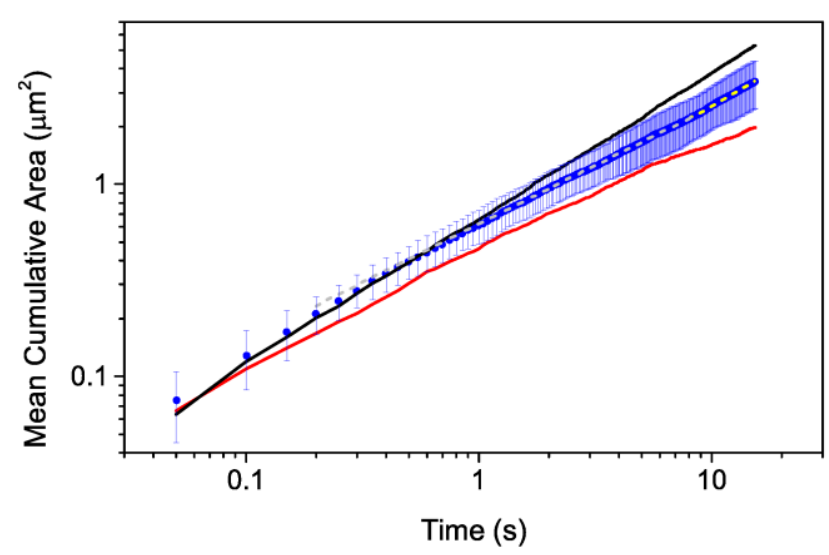

b

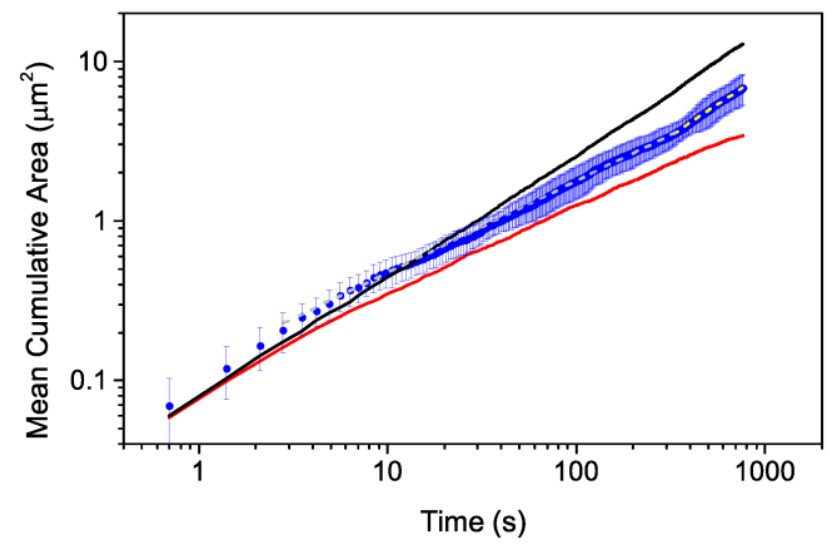

d

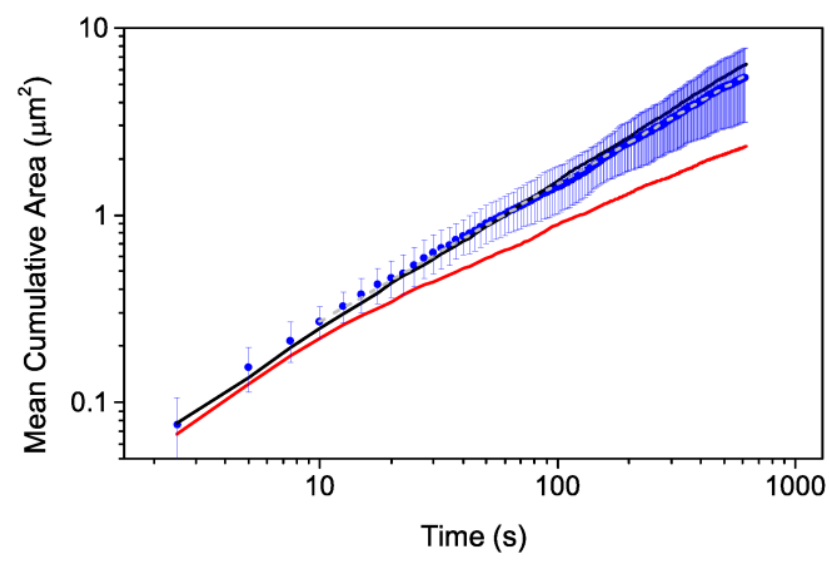

Figure 3. Mean cumulative area plots. The simulated mean cumulative area plots of 1D (red lines) and 2D (black lines) random motion and the experimentally obtained mean cumulative area plots for the $42 \mathrm{kbp}$ DNA (blue circles); (a) Dilute L (top) and Dilute C (bottom), (b) L-L, (c) C-C, and (d) C-L. The grey dashed lines are the fittings of the experimentally obtained plots by a power-law function. The grey and yellow dashed lines for L-L and C-C are the fittings of the two diffusion modes observed at different time scales with a power-law function. 


\section{Topology dependent diffusion rates of entangled chains.}

The diffusion coefficients of the long DNA molecules were determined by the initial slope of the cumulative area difference plot (see Experimental Section, Figs. 4a, 4b, and S7) while the relaxation times of the chains were determined by calculating an autocorrelation curve of the time-dependent fluctuation of the area occupied by the molecule (see Experimental Section, Figs. $4 \mathrm{~d}, 4 \mathrm{e}$, and S8). The mean diffusion coefficients $(D)$, the distribution of $D\left(\sigma_{D, \text { ex }} / \sigma_{D, \text { sim }}\right)$, and the mean relaxation time of the chains $\left(\tau_{\mathrm{r}}\right)$ are shown in Figures $4 \mathrm{c}$ and $4 \mathrm{f}$ (see also Figs. S9 and S10). The simultaneous determination of the accurate diffusion rate and conformational relaxation of the entire chain, which is possible only by using CA tracking, provides a unique technical platform on which to characterize the complicated dynamics of topological polymers. Note that the accurate diffusion rate cannot be obtained by conventional SMLT analysis (Fig. S11). The mean diffusion coefficient of Dilute $C\left(D_{\mathrm{C}}=1.10 \mu \mathrm{m}^{2} \mathrm{~s}^{-1}\right)$ is slightly faster than that of Dilute $\mathrm{L}\left(D_{\mathrm{L}}=0.99 \mu \mathrm{m}^{2} \mathrm{~s}^{-1}\right)$ as the cyclic molecule is smaller than its linear counterpart in the dilute solution (Figs. $4 \mathrm{a}, 4 \mathrm{c}$ top). The mean relaxation times of Dilute $\mathrm{C}\left(\tau_{\mathrm{rC}}=0.12 \mathrm{~s}\right)$ and Dilute $\mathrm{L}\left(\tau_{\mathrm{rL}}=0.14 \mathrm{~s}\right)$ determined by CA tracking are consistent with the slowest relaxation time of the dsDNA with similar lengths and topologies (Figs. 4d and 4f). ${ }^{70}$ These results confirm the validity of the use of CA tracking for accurate determination of the diffusion coefficient and the relaxation time. 
a

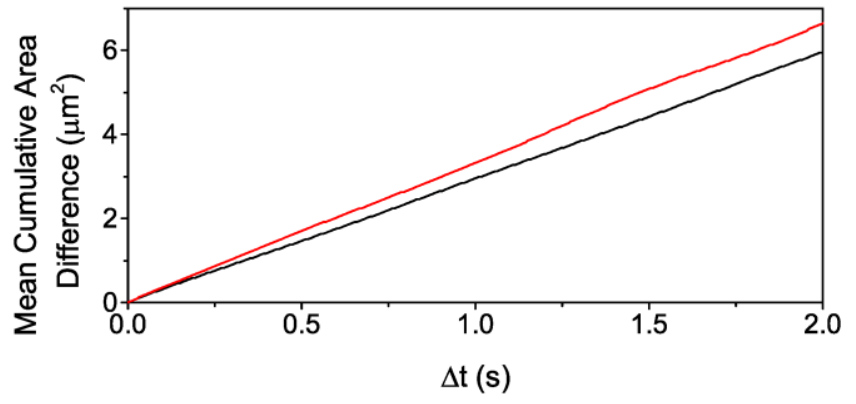

b

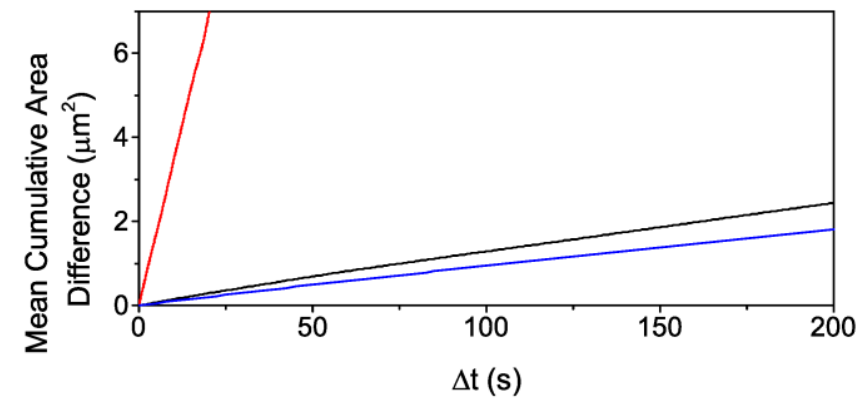

C

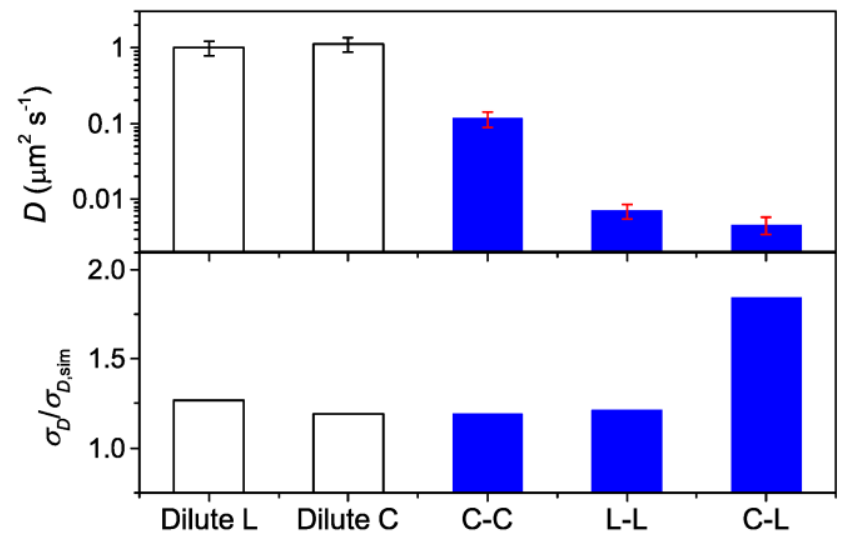

d

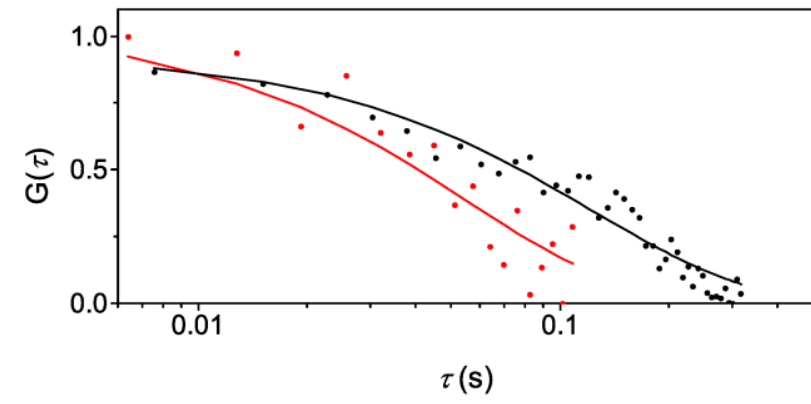

e

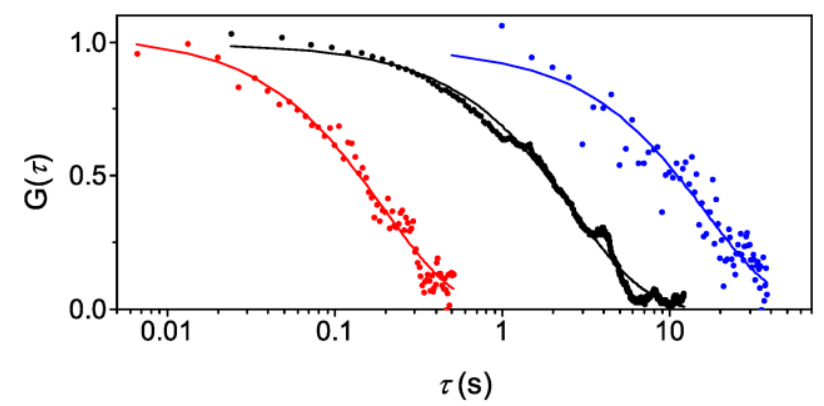

f

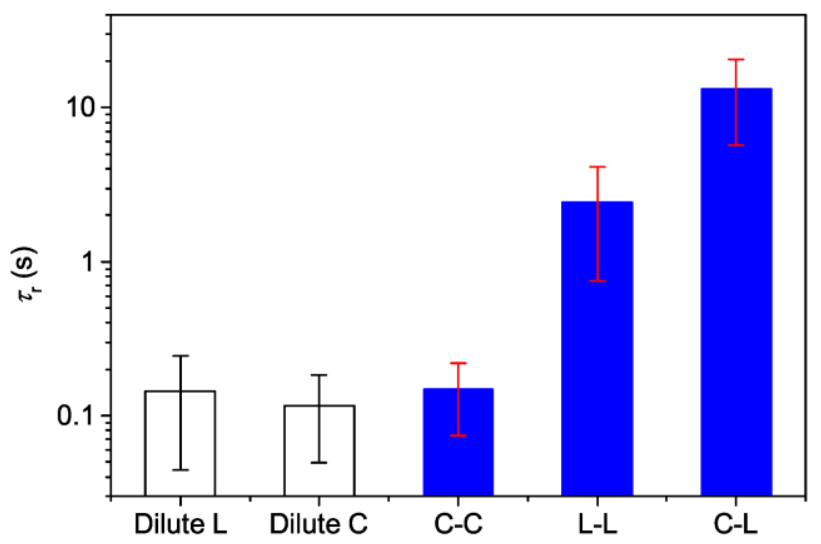

Figure 4. Cumulative area analysis of the diffusion and relaxation time. Typical cumulative area difference plots obtained for (a) Dilute L (black line) and Dilute C (red line) and (b) L-L (black line), C-C (red line), and C-L (blue line). (c) Mean diffusion coefficients (top) and the widths of their distribution (bottom) of Dilute L, Dilute C, L-L, C-C, and C-L determined by the initial slopes of the cumulative area difference plots. Typical autocorrelation curves of the timedependent fluctuation of the area occupied by (d) Dilute L (black line) and Dilute C (red line), 
and (e) L-L (black line), C-C (red line), and C-L (blue line). The solid lines show the fitting with a single-exponential decaying function. (f) Mean relaxation times of Dilute L, Dilute C, L-L, and C-C determined by the single-exponential fitting of the autocorrelation plots.

The topology of the polymer chain has a significant impact on the diffusion coefficient in the entangled solution. The mean diffusion coefficient of C-C $\left(D_{\mathrm{CC}}=0.11 \mu \mathrm{m}^{2} \mathrm{~s}^{-1}\right)$ is significantly faster than that of L-L $\left(D_{\mathrm{LL}}=0.0072 \mu \mathrm{m}^{2} \mathrm{~s}^{-1}\right)$ and C-L $\left(D_{\mathrm{CL}}=0.0046 \mu \mathrm{m}^{2} \mathrm{~s}^{-1}\right)$ (Fig. $4 \mathrm{c}$ top), consistent with a previous study. ${ }^{48}$ The diffusion of L-L is described by the reptation model given that the MCA plot of the $42 \mathrm{kbp}$ L-L shows 1D random motion of the chain at the length scale of the molecule. Indeed, both the $D_{\mathrm{LL}}$ and the slope of the MCA plot obtained by CA tracking are consistent with the previous observations, suggesting that the motion of the linear dsDNA with similar lengths in the entangled solution follows the reptation model. ${ }^{49}$ In contrast, the diffusion of $\mathrm{C}-\mathrm{C}$ cannot be described as the reptation motion. According to the reptation model, $D$ is inversely proportional to the squared length of the polymer chain. If C-C exhibits reptation motion, $D_{\mathrm{CC}}$ should be four times larger than $D_{\mathrm{LL}}$ as the effective chain length of the cyclic polymer is two times shorter than that of its linear counterpart. However, $D_{\mathrm{CC}}$ is more than an order of magnitude larger than $D_{\text {LL. }}$ Furthermore, the MCA plot of C-C shows clear deviation from the reptation model (i.e., 1D random motion at the length scale of the molecule, Fig. 3c). The MCA plot also shows deviation from the lattice-animal model (i.e., 2D random motion at the length scale of the molecule, Fig. 3c). Our results therefore suggest that the motion of C-C cannot be interpreted by existing models that are based on the concept of a chain moving through fixed obstacles (see below). 
The experimentally obtained $D_{\mathrm{C}-\mathrm{L}}$ is smaller than $D_{\mathrm{L}-\mathrm{L}}$. This is consistent with a previous observation $^{18,48}$ that was attributed to the threading of linear chains through the cyclic chain. A simulation study also predicts that the diffusion rate of cyclic molecules threaded by linear chains decreases significantly. ${ }^{71}$ Considering the multiple entanglements per chain in L-L (see above), we suggest that each cyclic molecule is threaded by multiple linear chains in C-L. The multiple threading should result in isotropic motion of the cyclic molecule. The 2D random motion observed for C-L provides strong evidence that the motion of the cyclic chain in C-L is indeed governed by threading. Importantly, $D_{\mathrm{C}-\mathrm{L}}$ has a broader distribution as compared with $D_{\mathrm{L}}$, $D_{\mathrm{C}}$, and $D_{\mathrm{L}-\mathrm{L}}$ (see Experimental Section, Fig. 4c bottom) whose distributions are captured by the statistical error of CA tracking (i.e., constant diffusion coefficients for all the molecules) (Fig. S12). The result is consistent with the large variations in the cumulative area plots obtained from different molecules in C-L compared with the other conditions (Fig. S6). It should be emphasized that such heterogeneity can be detected only through an accurate determination of the diffusion coefficient of the individual molecules by CA tracking, which is difficult with the conventional SMLT method due to the artificial broadening of the $D$ distribution (Fig. S11). Our previous studies on synthetic topological polymers also revealed such heterogeneity ${ }^{17,46}$ whereas these observations cannot be fully interpreted by the polymer physics theory that was developed based on ensemble-averaged experimental methods. This result clearly demonstrates the importance of visualizing and accurately analyzing the motion of individual molecules to unravel the molecular mechanism of the entanglement between topological polymers. The observed broader $D$ distribution might suggest that the diffusion rate is dependent on the threading number. ${ }^{46}$ Although the entanglement number in L-L should also vary between the chains, such broadening of the distribution is not observed (Fig. 4c bottom). These results point to the 
importance of considering molecular-level heterogeneity in the topological interaction between the chains in considering their diffusive motion, especially for inhomogeneous systems such as the mixture of linear and cyclic chains.

\section{Topology dependent relaxation modes of entangled chains.}

The topology of a polymer chain has a large effect on the relaxation time of the molecule. The relaxation time of C-C $\left(\tau_{\mathrm{rCC}}=0.15 \mathrm{~s}\right)$ is significantly shorter than that of $\mathrm{L}-\mathrm{L}\left(\tau_{\mathrm{rLL}}=2.44 \mathrm{~s}\right)$ and

$\mathrm{C}-\mathrm{L}\left(\tau_{\mathrm{rCL}}=13.2 \mathrm{~s}\right)($ Fig. $4 \mathrm{f})$. While it is not entirely clear if the relaxation time obtained for L-L corresponds directly to the reptation time of the molecule, a similar relaxation time that follows the scaling law for reptation of a polyelectrolyte has been reported for a dsDNA with similar length in an entangled solution. ${ }^{65}$ Thus, the relaxation time obtained for L-L by CA tracking directly reflects the conformational relaxation of the entire chain due to reptation motion. The relaxation time of $\mathrm{C}-\mathrm{C}$, on the other hand, deviates from the reptation model. According to the reptation model, $\tau_{\mathrm{r}}$ is proportional to the cube of the polymer chain length. ${ }^{3}$ The experimentally determined relaxation time for $\mathrm{C}-\mathrm{C}\left(\tau_{\mathrm{rCC}}=0.15 \mathrm{~s}\right)$ is much shorter than that predicted for the double-folded reptation model. More importantly, $\tau_{\mathrm{rCC}}$ is close to the relaxation time of Dilute $\mathrm{C}$ $\left(\tau_{\mathrm{rC}}=0.12 \mathrm{~s}\right)$. This observation clearly demonstrates that the relaxation is not affected by the interaction between the polymer chains in $\mathrm{C}-\mathrm{C}$ although the diffusive motion of the entire chain slows down by a factor of ten due to chain interactions (Fig. 4c top). This is in stark contrast to $\mathrm{L}-\mathrm{L}\left(\tau_{\mathrm{rLL}}=2.44 \mathrm{~s}\right)$ whose relaxation time is approximately 17 times slower than that of Dilute $\mathrm{L}$ $\left(\tau_{\mathrm{r} L}=0.14 \mathrm{~s}\right)$. These results together with the diffusion mode (Fig. 3c) and the rate (Fig. $4 \mathrm{c}$ top) suggest that the traditional concept of entanglement based on the single chain models (i.e., double-folded reptation and lattice-animal model) is not valid for cyclic polymers. Such a fast 
relaxation time cannot be accounted for without considering mutual relaxation of interacting chains (Fig. 5). Interestingly, the decoupling of the diffusive motion from the relaxation observed for the cyclic chain in C-C (Figs. 4c and 4f) has been predicted in a recent simulation study. ${ }^{72}$ The less interpenetrated chains in $\mathrm{C}-\mathrm{C}$ that were predicted by simulation could be partly responsible for the decoupling of the diffusive motion and relaxation.
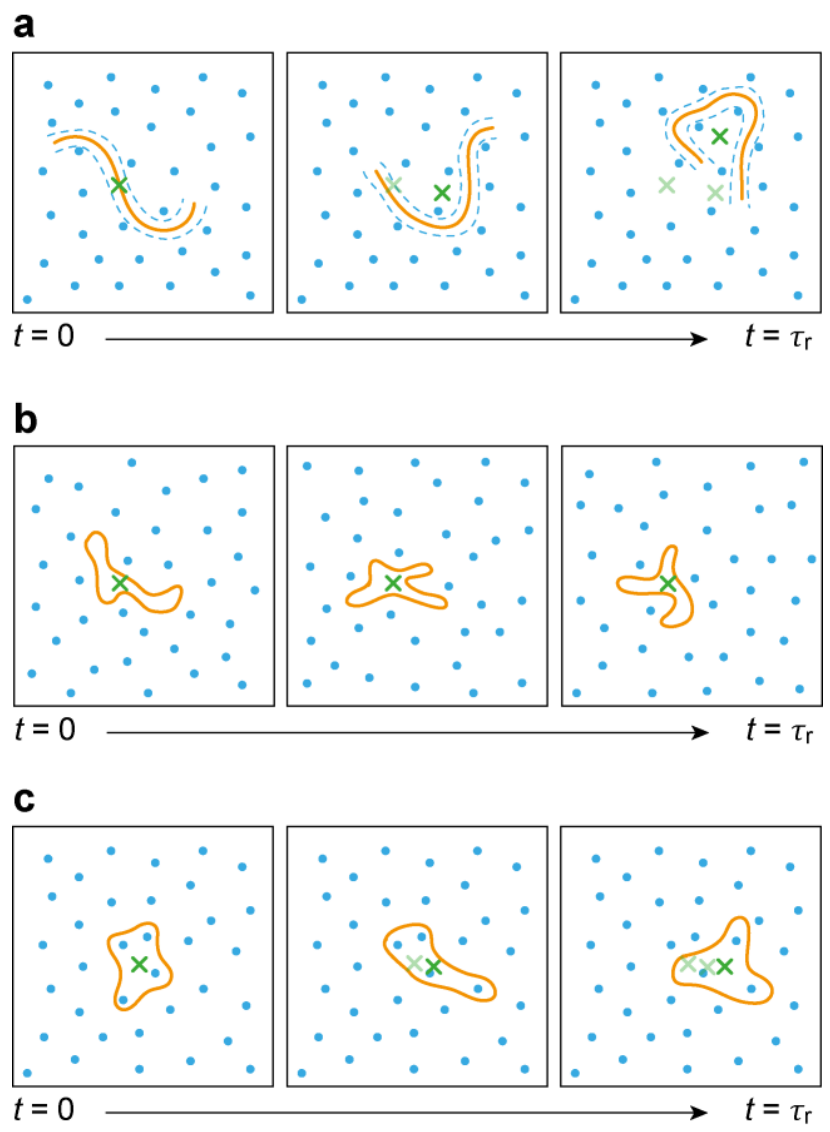

Figure 5. Schematic illustration of topology-dependent diffusion and relaxation. The motion and conformational relaxation of (a) L-L, (b) C-C, and (c) C-L (orange lines) occurring within their relaxation times. The cyan dots show obstacles due to the surrounding chains. The crosses show the center-of-mass at each time point. 
The relaxation time of C-L $\left(\tau_{\mathrm{rCL}}=13.2 \mathrm{~s}\right)$ is slower than that of $\mathrm{L}-\mathrm{L}\left(\tau_{\mathrm{rLL}}=2.44 \mathrm{~s}\right)$. Since the relaxation of L-L occurs via reptation motion (Fig. 3b), the $\tau_{\text {rLL }}$ captures the time that the chain takes to escape from the virtual tube created by the surrounding molecules. In contrast, the cyclic molecule in C-L cannot relax via reptation motion as the cyclic molecule is threaded by linear chains (Fig. 3d). Several models based on the constraint release have been proposed for the motion of the cyclic chain in the linear entangled solution and melt. ${ }^{67,71,73,74}$ The slower relaxation time and diffusion rate of $\mathrm{C}$-L observed experimentally in this study are consistent with those models. The result also indicates that the time scale of the constraint release motion can be experimentally estimated by our approach.

\section{CONCLUSIONS}

In conclusion, the motion of a cyclic polymer in an entangled solution was investigated at the single-molecule level by a newly developed cumulative-area (CA) tracking method. The direct visualization of the individual chains together with the simultaneous analysis of the diffusion mode, diffusion rate, and relaxation time by the CA tracking was shown to be remarkably effective for the quantitative characterization of the mode of the diffusive motion of long semiflexible polymers under entangled conditions. Our results point to the critical role of the mutual relaxation of the entangled chains in the motion of a cyclic polymer, underscoring the necessity to develop a new model to describe the motion of cyclic polymers under entangled conditions based on the mutual relaxation of the chains. The detailed characterization of topological polymer dynamics under entangled conditions at the single chain level has been possible only through simulation studies so far. CA tracking opens a unique opportunity to investigate polymer dynamics at the molecular level experimentally. Thermodynamic parameters related to diffusive 
and conformational dynamics can be determined separately by CA tracking. In addition, superresolution fluorescence imaging, which enables direct visualization of exact entangled points along the chain and therefore enables the direct comparison between the experiment and theory (i.e., primitive path analysis), is essentially compatible with CA tracking. Experiments are currently being conducted that will further unravel the controversial molecular mechanism of entanglement between topological polymers.

\section{ASSOCIATED CONTENT}

Supporting Information. Experimental details, details of the CA tracking analysis, simulated diffusion trajectories and cumulative-area images of 1D and 2D random motions, cumulative area plots obtained for simulated trajectories, an example of the CA tracking analysis for the calculation of the diffusion coefficient, and example of the CA tracking analysis for the calculation of the relaxation time, frequency histograms of the diffusion coefficients and relaxation times, comparison between the $\mathrm{CA}$ tracking analysis and the localization-tracking analysis on the determination of the diffusion coefficients, comparison between the simulated and experimentally obtained histograms of the diffusion coefficients. This material is available free of charge via the Internet at http://pubs.acs.org.

\section{AUTHOR INFORMATION}

\section{Corresponding Author}

*(S.H.) satoshi.habuchi@kaust.edu.sa

\section{Author Contributions}

$\$$ These authors contributed equally. 


\section{Notes}

The authors declare no competing financial interest.

\section{ACKNOWLEDGMENT}

The research reported in this publication was supported by King Abdullah University of Science and Technology.

\section{REFERENCES}

(1) De Gennes, P.-G., Scaling Concepts in Polymer Physics. Cornell University Press: 1979.

(2) Doi, M.; Edwards, S. F., The Theory of Polymer Dynamics. Oxford University Press: 1986.

(3) Rubinstein, M.; Colby, R. H., Polymer Physics. Oxford University Press: 2003.

(4) Auhl, R.; Everaers, R.; Grest, G. S.; Kremer, K.; Plimpton, S. J., J. Chem. Phys. 2003, 119, $12718-12728$.

(5) Vettorel, T.; Grosberg, A. Y.; Kremer, K., Phys. Biol. 2009, 6, 10.

(6) In Topological Polymer Chemistry: Progress of Cyclic Polymers in Syntheses, Properties and Functions, Tezuka, Y., Ed. World Scientific Publishing Co. Pte. Ltd.: Singapore, 2013; pp 137-156.

(7) Honda, S.; Yamamoto, T.; Tezuka, Y., J. Am. Chem. Soc. 2010, 132, 10251-10253.

(8) Honda, S.; Yamamoto, T.; Tezuka, Y., Nat. Commun. 2013, 4, 1574.

(9) Bohn, M.; Heermann, D. W., PLoS One 2010, 5, 14.

(10) Cook, P. R.; Marenduzzo, D., J. Cell Biol. 2009, 186, 825-834. 
(11) Jun, S.; Mulder, B., Proc. Natl. Acad. Sci. U. S. A. 2006, 103, 12388-12393.

(12) Rosa, A.; Everaers, R., PLoS Comput. Biol. 2008, 4, e1000153.

(13) Sachs, R. K.; Vandenengh, G.; Trask, B.; Yokota, H.; Hearst, J. E., Proc. Natl. Acad. Sci. U. S. A. 1995, 92, 2710-2714.

(14) McLeish, T., Science 2002, 297, 2005-2006.

(15) McLeish, T., Nat. Mater. 2008, 7, 933-935.

(16) Hur, K.; Jeong, C.; Winkler, R. G.; Lacevic, N.; Gee, R. H.; Yoon, D. Y., Macromolecules 2011, 44, 2311-2315.

(17) Habuchi, S.; Satoh, N.; Yamamoto, T.; Tezuka, Y.; Vacha, M., Angew. Chem. Int. Ed. 2010, 49, 1418-1421.

(18) Nam, S.; Leisen, J.; Breedveld, V.; Beckham, H. W., Macromolecules 2009, 42, 31213128.

(19) Cosgrove, T.; Griffiths, P. C.; Hollingshurst, J.; Richards, R. D. C.; Semlyen, J. A., Macromolecules 1992, 25, 6761-6764.

(20) Kawaguchi, D.; Masuoka, K.; Takano, A.; Tanaka, K.; Nagamura, T.; Torikai, N.; Dalgliesh, R. M.; Langridge, S.; Matsushita, Y., Macromolecules 2006, 39, 5180-5182.

(21) Pasquino, R.; Vasilakopoulos, T. C.; Jeong, Y. C.; Lee, H.; Rogers, S.; Sakellariou, G.; Allgaier, J.; Takano, A.; Bras, A. R.; Chang, T.; Goossen, S.; Pyckhout-Hintzen, W.; Wischnewski, A.; Hadjichristidis, N.; Richter, D.; Rubinstein, M.; Vlassopoulos, D., ACS Macro Lett. 2013, 2, 874-878. 
(22) Roovers, J., Macromolecules 1985, 18, 1359-1361.

(23) von Meerwall, E.; Ozisik, R.; Mattice, W. L.; Pfister, P. M., J. Chem. Phys. 2003, 118, 3867-3873.

(24) Brown, S.; Szamel, G., J. Chem. Phys. 1998, 109, 6184-6192.

(25) Muller, M.; Wittmer, J. P.; Cates, M. E., Phys. Rev. E 1996, 53, 5063-5074.

(26) Reigh, S. Y.; Yoon, D. Y., ACS Macro Lett. 2013, 2, 296-300.

(27) Suzuki, J.; Takano, A.; Deguchi, T.; Matsushita, Y., J. Chem. Phys. 2009, 131, 144902.

(28) Roovers, J., Overview on physical properties of cyclic polymers. In Topological Polymer Chemistry: Progress of Cyclic Polymers in Syntheses, Properties and Functions, Tezuka, Y., Ed. World Scientific Publishing Co. Pte. Ltd.: Singapore, 2013; pp 137-156.

(29) Klein, J., Nature 1978, 271, 143-145.

(30) Leger, L.; Hervet, H.; Rondelez, F., Macromolecules 1981, 14, 1732-1738.

(31) von Meerwall, E. D.; Amis, E. J.; Ferry, J. D., Macromolecules 1985, 18, 260-266.

(32) Aoki, H.; Mori, K.; Ito, S., Soft Matter 2012, 8, 4390-4395.

(33) Gavranovic, G. T.; Csihony, S.; Bowden, N. B.; Hawker, C. J.; Waymouth, R. M.; Moerner, W. E.; Fuller, G. G., Macromolecules 2006, 39, 8121-8127.

(34) Muls, B.; Uji-i, H.; Melnikov, S.; Moussa, A.; Verheijen, W.; Soumillion, J. P.; Josemon, J.; Mullen, K.; Hofkens, J., ChemPhysChem 2005, 6, 2286-2294.

(35) Skaug, M. J.; Mabry, J. N.; Schwartz, D. K., J. Am. Chem. Soc. 2014, 136, 1327-1332. 
(36) Yu, C. Q.; Guan, J.; Chen, K. J.; Bae, S. C.; Granick, S., ACS Nano 2013, 7, 9735-9742.

(37) Bartko, A. P.; Xu, K. W.; Dickson, R. M., Phys. Rev. Lett. 2002, 89.

(38) Deres, A.; Floudas, G. A.; Mullen, K.; Van der Auweraer, M.; De Schryver, F.; Enderlein, J.; Uji-i, H.; Hofkens, J., Macromolecules 2011, 44, 9703-9709.

(39) Flier, B. M. I.; Baier, M. C.; Huber, J.; Mullen, K.; Mecking, S.; Zumbusch, A.; Woll, D., J. Am. Chem. Soc. 2012, 134, 480-488.

(40) Habuchi, S.; Oba, T.; Vacha, M., Phys. Chem. Chem. Phys. 2011, 13, 6970-6976.

(41) Oba, T.; Vacha, M., ACS Macro Lett. 2012, 1, 784-788.

(42) Schob, A.; Cichos, F.; Schuster, J.; von Borczyskowski, C., Eur. Polym. J. 2004, 40, 10191026.

(43) Uji-i, H.; Melnikov, S. M.; Deres, A.; Bergamini, G.; De Schryver, F.; Herrmann, A.; Mullen, K.; Enderlein, J.; Hofkens, J., Polymer 2006, 47, 2511-2518.

(44) Woll, D.; Braeken, E.; Deres, A.; De Schryver, F. C.; Uji-i, H.; Hofkens, J., Chem. Soc. Rev. 2009, 38, 313-328.

(45) Habuchi, S.; Fujiwara, S.; Yamamoto, T.; Tezuka, Y., Polym. Chem. 2015, 6, 4109-4115.

(46) Habuchi, S.; Fujiwara, S.; Yamamoto, T.; Vacha, M.; Tezuka, Y., Anal. Chem. 2013, 85, 7369-7376.

(47) Perkins, T. T.; Smith, D. E.; Chu, S., Science 1994, 264, 819-822.

(48) Robertson, R. M.; Smith, D. E., Proc. Natl. Acad. Sci. U. S. A. 2007, 104, 4824-4827. 
(49) Smith, D. E.; Perkins, T. T.; Chu, S., Phys. Rev. Lett. 1995, 75, 4146-4149.

(50) Robertson, R. M.; Smith, D. E., Macromolecules 2007, 40, 3373-3377.

(51) Perkins, T. T.; Quake, S. R.; Smith, D. E.; Chu, S., Science 1994, 264, 822-826.

(52) Quake, S. R.; Babcock, H.; Chu, S., Nature 1997, 388, 151-154.

(53) Cohen, A. E.; Moerner, W. E., Proc. Natl. Acad. Sci. U. S. A. 2006, 103, 4362-4365.

(54) Cohen, A. E.; Moerner, W. E., Phys. Rev. Lett. 2007, 98, 116001.

(55) Lumma, D.; Keller, S.; Vilgis, T.; Radler, J. O., Phys. Rev. Lett. 2003, 90, 218301.

(56) Petrov, E. P.; Ohrt, T.; Winkler, R. G.; Schwille, P., Phys. Rev. Lett. 2006, 97, 258101.

(57) Schaeffel, D.; Yordanov, S.; Staff, R. H.; Kreyes, A.; Zhao, Y.; Schmidt, M.; Landfester, K.; Hofkens, J.; Butt, H. J.; Crespy, D.; Koynov, K., ACS Macro Lett. 2015, 4, 171-176.

(58) Zettl, U.; Hoffmann, S. T.; Koberling, F.; Krausch, G.; Enderlein, J.; Harnau, L.; Ballauff, M., Macromolecules 2009, 42, 9537-9547.

(59) Serag, M. F.; Abadi, M.; Habuchi, S., Nat. Commun. 2014, 5, 5123.

(60) Robertson, R. M.; Laib, S.; Smith, D. E., Proc. Natl. Acad. Sci. U. S. A. 2006, 103, 73107314.

(61) Kapnistos, M.; Lang, M.; Vlassopoulos, D.; Pyckhout-Hintzen, W.; Richter, D.; Cho, D.; Chang, T.; Rubinstein, M., Nat. Mater. 2008, 7, 997-1002.

(62) Hellriegel, C.; Kirstein, J.; Brauchle, C., New J. Phys. 2005, 7, 23. 
(63) Tree, D. R.; Muralidhar, A.; Doyle, P. S.; Dorfman, K. D., Macromolecules 2013, 46, 8369-8382.

(64) Kroger, M.; Hess, S., Physica A 1993, 195, 336-353.

(65) Zhu, X. Y.; Kundukad, B.; van der Maarel, J. R. C., J. Chem. Phys. 2008, 129, 185103.

(66) Teixeira, R. E.; Dambal, A. K.; Richter, D. H.; Shaqfeh, E. S. G.; Chu, S., Macromolecules 2007, 40, 2461-2476.

(67) Klein, J., Macromolecules 1986, 19, 105-118.

(68) Milner, S. T.; Newhall, J. D., Phys. Rev. Lett. 2010, 105, 208302.

(69) Obukhov, S. P.; Rubinstein, M.; Duke, T., Phys. Rev. Lett. 1994, 73, 1263-1266.

(70) McHale, K.; Mabuchi, H., J. Am. Chem. Soc. 2009, 131, 17901-17907.

(71) Yang, Y. B.; Sun, Z. Y.; Fu, C. L.; An, L. J.; Wang, Z. G., J. Chem. Phys. 2010, 133, 064901.

(72) Halverson, J. D.; Lee, W. B.; Grest, G. S.; Grosberg, A. Y.; Kremer, K., J. Chem. Phys. 2011, 134, 204905.

(73) Halverson, J. D.; Grest, G. S.; Grosberg, A. Y.; Kremer, K., Phys. Rev. Lett. 2012, $108,5$.

(74) Mills, P. J.; Mayer, J. W.; Kramer, E. J.; Hadziioannou, G.; Lutz, P.; Strazielle, C.; Rempp, P.; Kovacs, A. J., Macromolecules 1987, 20, 513-518. 
For Table of Contents use only

Single-molecule imaging reveals topology dependent mutual relaxation of polymer chains

Maram Abadi ${ }^{\ddagger}$, Maged F. Serag ${ }^{\ddagger}$, and Satoshi Habuchi*

TOC graphic

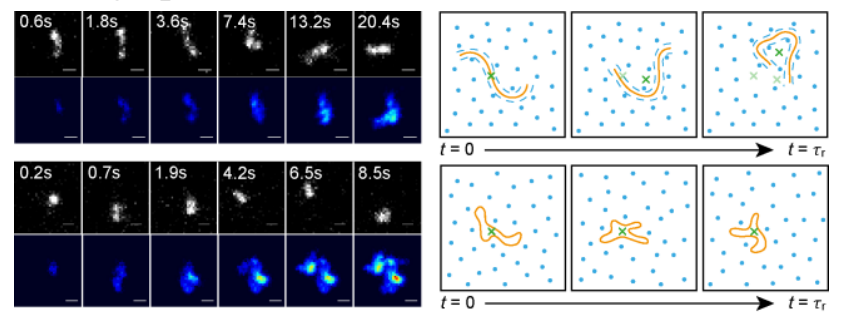

